\title{
NEOREG: Design and implementation of an online Neonatal \\ Registration System to access, follow and analyse data of new- borns with congenital Cytomegalovirus infection
}

\author{
Kristof Steurbaut ${ }^{1}$, Femke De Backere ${ }^{1}$, Annelies Keymeulen ${ }^{2}$, Marc De Leenheer ${ }^{1}$, Koenraad \\ Smets ${ }^{2}$, Filip De Turck $^{1}$ \\ ${ }^{1}$ Department of Information Technology, Ghent University-IBBT, Gaston Crommenlaan 8 bus 201, 9050 Ghent, Belgium \\ 2 Department of Neonatology, Ghent University Hospital, De Pintelaan 185, 9000 Ghent, Belgium
}

\begin{abstract}
Summary
Today's registration of newborns with congenital cytomegalovirus (cCMV) infection is still performed on paper-based forms in Flanders, Belgium. This process has a large administrative impact. It is important that all screening tests are registered to have a complete idea of the impact of cCMV. Although these registrations are usable in computerized data analysis, this data is not available in a format to perform electronic processing. An online Neonatal Registry (NEOREG) System was designed and developed to access, follow and analyse data of newborns remotely. It allows remote access and monitoring by the physician. The Java Enterprise layered application provides patients' diagnostic registration and treatment follow-up through a web interface and using document forms in Portable Document Format (PDF) which incorporate all elements from existing forms. Forms are automatically processed to structured EHRs. Modules are included to perform statistical analysis. The design was driven by extendibility, security and usability requirements. The website load time, throughput and execution time of data analysis were evaluated in detail. The NEOREG system is able to replace the existing paper-based CMV records.
\end{abstract}

\section{Keywords}

Health informatics, web-based registration system, congenital cytomegalovirus registry

\section{Correspondence to:}

Kristof Steurbaut

Ghent University - IBBT

Department of Information Technology

Gaston Crommenlaan 8 bus 201

9050 Gent, Belgium

Phone: +32 9 3314940, Fax: +32 93314899

E-mail: kristof.steurbaut@intec.ugent.be 


\section{Introduction}

The Flemish Society of Paediatrics' Neonatology and Perinatal Epidemiology Working Group aims to provide guidelines concerning diagnosis and therapy of new-borns infected with congenital cytomegalovirus (cCMV) and to manage the follow-up of those children with the aim of studying the outcome prediction of the disease [1]. The guidelines provide physicians with evidence-based information and recommendations to treat patients with CCMV. The working group consists of neonatologists, otorhinolaryngologists and general paediatricians in Flanders. In eight Flemish hospitals, equipped with a neonatal intensive care unit (NICU), paper-based registration forms for CCMV infection have already been used since January 2007, with the purpose to register as much new-borns as possible with CCMV and track their progress.

Cytomegalovirus is the most common cause of congenital infection [2]. The incidence varies worldwide with an incidence of 0.5 to $1.3 \%$ of all live born infants in Flanders. With an annual birth number of 65,000-70,000 new-borns in Flanders [3] this means that about 400-600 new-borns are affected per year. cCMV is the leading cause of sensor neural hearing loss, an important cause of neurologic developmental delay and causes a great disease burden on child, parent and community. The infection is transmitted prenatally. $10 \%$ of the infected new-borns are symptomatic at birth, with symptoms being various, including hepatitis, low platelet count, microcephaly, intra-uterine growth restriction, hepatosplenomegaly or convulsions. Most of the infected new-borns (85-90\%) have no clinical symptoms at birth. It is important that all of the infected children undergo screening tests (blood analysis, MRI and ultrasound of the brain, ophthalmologic and hearing evolution) to evaluate the impact of cCMV. Only the children, who are symptomatic at birth or have aberrant screening tests, may benefit from treatment with Ganciclovir (Cymevene) [4]. When an infant fails the hearing test, the baby is retested with at least one week interval. When failing is confirmed, the baby is referred to the audiology department for further assessment with brainstem evoked response audiometry. When hearing loss is confirmed, therapy with (val)ganciclovir is discussed with the parents [5]. However, the asymptomatic children are still at risk to develop long term sequel over the next years. These sequelae include hearing problems, neurodevelopmental delay and visual impairment. For that reason, it is suggested that all children with cCMV are followed during several years, so pending neurological, hearing, and visual problems can be detected in time.

The Neonatology departments of the academic hospitals of Ghent, Antwerp, Louvain and regional hospitals in Bruges, Antwerp, Wilrijk, Ghent perform a detailed registration of cCMV. For example, the NICU of the Ghent University Hospital consists of 32 beds, and acts as the coordinating study centre. Collecting patients' data has the potential to reveal correlations between diagnostic variables, 
offer new insights in the data and hence lead to better treatment and improved quality of care. However, today, this data is still collected manually in paper forms, making it very difficult to perform data mining or apply computing techniques to discover trends.

When $\mathrm{CCMV}$ is detected in the laboratory results of the new-borns' urine, the physician fills out a paper-based form, after informed consent of the parents. The parents' permission is asked to record the data in an electronic register and data is anonymized for data research. Figure 1 shows schematically the current workflow. The diagnosis forms (1) include the results of central radiology (based on echography, CT scan or NMR scan), eye fundus, audio logical results and blood analysis. A follow-up process has to be completed with audio logic evaluation each 3 months till the age of 3 years and later each year till the age of 6 years. The neurological development is evaluated at 4 months, 1 year, 1.5 years, 4.5 years and 6 years. The ophthalmologic follow up is repeated every year. All paper forms are delivered to the coordinating centre (2). Current analysis of the paperbased records is time-consuming, labour-intensive and difficult. At the coordinating hospital, the content of the forms is entered in a computerized spread sheet (3) to enable minimal data analysis (4). For long-term statistical and pattern analysis, a database is needed. It is important that the system supports flexible updates when new data types or decision support facilities are needed.

The potential of electronic records to conduct data analysis and support clinical decisions has already been widely recognized [6-8]. However, data is often not available in a format for analysis and research [9]. Computerized decision support systems can be developed that estimate prognosis, detect patterns and as such support ethical decision making in complex medical issues for parents and physicians in NICU [10]. Yang et al. [10] address the challenging question if there are data indicators with respect to the childs' health status that can guide the best decisions regarding when to initiate, withhold or terminate treatment. Skouroliakou et al. [11] studied the benefits of an electronic registry in a NICU in Greece and found that electronic collection and analysis led to significant findings at epidemiological, medical resource utilization and hospital cost levels. According to Cordero et al. [12], the implementation of computerized physician order entry (CPOE) resulted in reduction in medication times, medication errors and decreases in service response times.

Electronic Health Records (EHR) facilitate better follow-up of physician's diagnosis and the patients' condition. Urschitz et al. [13] reported about the experience with a Patient Data Management System (PDMS) at a NICU in Vienna, Austria. The database gets manual input and inputs from the monitoring system, by collecting data from external data sources, and from the lab information system. Knowledge based systems are available for calculating the parenteral nutrition of newborn infants, for advising medication, for managing mechanical ventilation. More than $2 / 3$ of the users 
preferred computer assisted documentation to charting by hand. PDMS have to be constantly adapted to the user's needs and to the changing clinical environment. However the benefits of electronic registration, not all departments have already adopted an electronic register. Moreover, the $\mathrm{CCMV}$ registration is not included in existing clinical information systems.

In this paper we propose the web-based Neonatology Registration System (NEOREG) which supports data registration of $\mathrm{CMV}$ and automates the follow-up process. The system has the following objectives for registration of newborns: registration of (i) the physician's diagnosis, (ii) abnormalities at birth, (iii) evolution of treatment and (iv) the periodic follow-ups of psych motoric function, hearing and visas at long term (till the age of 6 years). NEOREG aims to provide a patient registration and remote follow-up system for all children infected with cytomegalovirus in Flanders, Belgium. Using NEOREG, human processing tasks are eliminated, as shown in Figure 1. The website offers statistical components to visualize patient's prognosis at long term. If a follow-up is necessary, an email alert is generated to arrange an appointment with the patient. All records can be accessed remotely on the secure website. In previous work $[14,15]$ we have already demonstrated the benefits of computerized support for the Intensive Care Unit (ICU) for the follow-up of infections and management of antibiotic therapies. In contrast to the latter research project, where integration with already existing electronic records from diverse sources was performed, the NEOREG System replaces the existing paper-based registrations of $\mathrm{cCMV}$.

\section{Paper Organization}

This article is structured as follows. The Methods section details the registration scenario. Design objectives for the architecture are described, together with a detailed explanation of the architecture. Next, implementation details are provided. Furthermore, a description is provided of the usage of the Java Enterprise framework, the PDF form generation process, persistence of data and data analysis. The Results section contains the dataset characteristics and the performance evaluation. The discussion section deals with the impact of electronic records versus paper-based records. Finally, in the conclusion the contributions to the registration of CCMV are summarized.

\section{Methods}

In this section the development methodology is described. First, the registration scenario with several questionnaires is described in detail. Second, the design objectives are formulated. In the architecture details, the layered application is shown. Finally, implementation details are presented. 


\section{Registration scenario}

After secure login, a newborn infected with CMV can be registered by uploading a filled-out questionnaire. The system starts a follow-up mechanism, in which a timely follow-up of the patient is requested, as an electronic alert to the responsible physician or paediatrician. Each data entry form is a Portable Document Format (PDF) file, as required by the infection working group in order to guarantee a higher adoption by physicians after a switch from the paper-based files. PDFs can be stored and filled-out on off line systems too. Only for the upload and access to historical patient records an Internet connection is required. The system converts the information in the PDF file to electronic records in a database. Figure 2 shows a screenshot of the registration form. There are six available CMV questionnaires in the system:

1. Registration form: this form consists of general information about the newborn. It includes detailed information about the birth and the mother.

2. Diagnosis form: the form contains the results of the examinations, which forms the basis for the physician's diagnosis. It has the results of examinations in prenatal and postnatal phase.

3. Treatment form: this form enables the physician to start or stop a therapy.

4. Follow-up Development form: periodic follow-up concerning the patient's condition, the mental state, $I Q$, psych motoric functioning, is registered.

5. Audio logical Diagnostics Intake form: the form includes several clinical evaluation results of the hearing of the patient.

6. Audio logical Diagnostics Follow-up form: the audio logical aspect is continuously followed. The form is filled out at the age of $3,6,12,18,24,30$ months and at least once per year till the age of 6 years.

\section{Design objectives}

The focus was on the security, usability and extendibility requirements. We applied the layered approach of enterprise applications. The three-layered architecture consists of a Presentation layer, Processing or Business layer and Data layer. The Presentation layer offers a registration website. Through a security component, access is given to the medical records according to the user's role and the hospital policy. The website enables the upload and download of forms. The Processing layer collects the information in the forms and instantiates the information in data objects. The Data layer provides the persistence of the objects in the database.

Security is enforced by a role-based authentication mechanism. This means that at login only access is given to functionality according to the role access privileges. NEOREG has three user roles: system administrator, physician or paediatrician, clinical researcher. The last role has only access to anonymous data analysis for clinical studies, while the physician can see all data. The system 
administrator has access to configuration of the system. Additionally, the registration and login of a user can be done with an electronic identity card or by user-password credentials. The Belgian identity card can be used for authentication and authorization. The PDF forms are also protected with the user's password.

The second objective is usability for registration, display and analysis of patients' data to stimulate adoption. The system must be integrated in the existing physician's workflow. Therefore, this electronic registration is almost identical as paper-based registration. Instead of the paper forms, data is entered in a PDF, which can be processed by a computer. If the physician still wants to keep a paper-based print-out, an identical paper will be produced, while the system stores the data entries for long term analysis. The system should offer a display with a summary of all entered data. In addition to simply storing the data, the system should provide data analysis functions (allowing for instance estimation of hearing prognosis of the patient or statistics of the population of newborns with cCMV).

A third architectural driver is extendibility of the registration. As the medical therapies are evolving, the registration forms should be extendable with new data entries. The existing template form contains a list of possible diagnostic options, treatment or measurement categories. This list can be adapted at runtime by changing the configuration. In fact, these configured options are added in the database, which enables the PDF template form to dynamically rebuild itself. The new empty template can be downloaded by the physician. Existing filled-out templates are still supported.

\section{Architecture Details}

The layered architecture is shown in Figure 3. The Web-Presentation layer with the web pages (Figure 4) has a Security component that ensures authorized access to the records. The Processing layer has specific components for the follow-up and analysis. The PDF processing component creates automatically new survey forms and processes the filled out forms. Initial questionnaire templates are configured and can automatically be updated in the web interface, if new questions should be added. E-mails and alerts of data changes, new registered users or platform maintenance are delivered by the Alerting component. A Timer component keeps track of the follow-up moments and sends automatically an e-mail to the physician to remind that a new consultation is needed. The periodic follow-up periods are preconfigured. The Analysis component includes 17 predefined analysis cases. For each case specific data queries are available. It can be used in statistical clinical research. The main purpose of the Data layer is to provide a complete storage of CMV records. The database consists of general information of the newborn and the mother. Diagnosis, follow-up, audio 
logical diagnose and audio logical follow-up data are linked to the patient data when a form is uploaded.

\section{Implementation of the web-based registration system}

Java Enterprise Application

The NEOREG system was developed using Java Enterprise Edition (Java EE) platform and deployed on the Glassfish Application Server. Java EE has already been applied in e-commerce [16], e-learning [17], as well as in e-health with real life cases at intensive care [18], dental care [19], and laboratory [20]. Its layered approach has several advantages [19]: (a) changes in database do not affect the user's application, (b) scalability or extendibility is provided by using a separate business layer, and (c) security mechanisms are provided by the platform.

Java Server Faces (JSF) technology is embedded in XHTML. Additional JSF component are used from the Primefaces library, for example to support AJAX calls. The role-based security is enforced by the Java Authentication and Authorization Service. The web layer has managed beans to call session beans. Language independence is enabled through property files. In the business layer session beans act as facade to the data layer or provide logic. Facade session beans have operations to create new entities, persist, remove, find and merge with existing data objects. The timer is implemented as an EJB Timer session bean and checks daily if new alerts for consultations are needed.

\section{Portable Questionnaire Forms}

The paper-based forms are replaced with Adobe Portable Document Format (PDF) forms. It has the advantage of being independent from the applications, hardware and operating system. The PDF files contain interactive elements such as form fields. In the NEOREG system we used the iText open source Java Library [21] to create and read the PDF forms. PDF Encryption is enabled at creation time. The PDF template form is built up from code and the input values for checkboxes and text fields are selected from the database. Since the PDF 1.2 specification, AcroForms or Acrobat Forms have been supported for integrating data and PDF forms. The AcroForms provide standard form components (such as text fields, radio buttons and checkboxes) and support limited Javascript usage for validation of input fields. The data elements are kept in key-value pairs. The PDF component uses iText to read data form entries and insert data in the database.

\section{Data Persistence}

The database (Figure 5) is built up through the Java EE Persistence API, a Java programming framework for the management of relational data. An entity maps to a table in the database, while 
instances of the entity correspond to individual rows in a table. Relationships between entities can be expressed in objects with metadata and annotations. Considering the hospital's experience, we used the Oracle 9 database.

One of the main objectives was the possibility to change and extend existing functionality and persisted data. During the development we investigated two persistence approaches to cope with changes in the data forms. One approach is to rebuild and automatically change data columns and tables when major changes are applied to the form templates. Each object (entity) contains annotations which guide the persistence process. For example the annotation Entity means that the object will be transformed into a data table. As such, relationships between objects and the actual objects are modelled at design time. When new templates are created, Java reflection is used to create the necessary Java programming code, which is later deployed on the server. The second approach is to maintain the current data structure, but change the configuration of data values. In this approach, additional data values are stored in the configuration table that builds up the form. By creating many-to-many relationships between data variables and form template, the form is automatically built up with the necessary data variables that should be included in the questionnaire. We chose the second approach for the system to minimize redeployment of the system.

\section{Results}

\section{Dataset Characteristics}

The current dataset of the NICU of Ghent University contains 149 patients. The working group consists of 20 members from the participating hospitals. The 149 patients comprise $74(49.7 \%)$ male newborns, 70 (47.0\%) female newborns and $5(3.4 \%)$ missing documented cases. The set spans the period from January 1, 2007 to December 31, 2010. The patients' parents approved the registration by signing an informed consent giving permission to include the newborns' data in the electronic database and to use the data for scientific purposes on the condition that confidentiality is ensured.

\section{Performance Results}

The performance was evaluated by emulating 500 simultaneous users interacting with the web application. We evaluated the performance by measuring the load times of the web frontend (as shown in Figure 6). Each user calls the website through an http request. The evaluation was performed on a client that has an Intel Core 2 Duo CPU with $2.4 \mathrm{GHz}$ and 4 GB RAM, running the 64bit Windows 7 Operating System (OS), Java Runtime 1.6.21 and Apache JMeter. The web application was deployed on a Windows XP Pro OS server on an AMD Athlon 64 X2 Dual Core Processor 5200+ 
with $2.7 \mathrm{GHz}$ and $3 \mathrm{~GB}$ RAM. The server has the Glassfish 3.0 Application Server. It is expected that initially only 10 to 50 users will access the application. This corresponds to a load time between 28 and 48 milliseconds, which results in an excellent quality of experience of the users. Figure 7 shows the throughput and number of kilobytes per second for 10 to 500 users. Table 1 shows the detailed results of a typical user scenario, measured by FireBug, a plug-in for web browser Firefox. The user logs in on the website with his/her user credentials, downloads an empty PDF questionnaire, fills in this template and uploads the form. Later the user reviews and analyses this data on the website. The display and download times are within acceptable range but the PDF takes more time due to the generation process. In addition, the execution times for the retrieval of data analysis cases were also measured. Figure 8 shows the execution times for the audio logic data and neurologic data for several data analysis cases. One case had a longer execution time and took $61.12 \mathrm{~s}$. All other cases' execution time ranges from 0.23 to 2.54 seconds and are shown on Figure 8. The queries for audio logical data take more time due to a higher number of variables in the queries.

\section{Discussion}

Today, most hospitals are equipped with an information system that monitors vital parameters and collects records from laboratory and observations. Unfortunately, the coordinating NICU was not using a complete system for registration of newborns' diagnosis. The NEOREG system fulfils the need for the registration of congenital cytomegalovirus infection and could even be extended to registration of other parameters by adding forms or integrating the outputs of medical devices directly in the system. The NEOREG system follows a centralized approach in which CCMV patient records of the hospitals are stored in a central database. In the paper-based registration it was noticed that there were a large number of missing registrations. Afterwards, it was not clear if the values were left intentionally blank on the paper forms or if part of the follow-up process was missing. Using a computerized registration, the completeness of the forms and the timely follow-ups can be validated automatically and users can automatically be reminded at regular times.

Christensen and Grimsmo [22] conducted a study of GPs' use of electronic patient records in terms of the use of different functionality, time spent and the effects of EHRs in Norway. The overall availability of individual patient records had improved compared to the handwritten paper-based records, but the information within the EHR was not satisfactory. Time studies on physician use of EHR didn't show a time reduction but the potential benefits were: simultaneous access by multiple users, improved readability compared to handwriting, automated generation of reports in different formats to do analysis. The proposed system also offers these benefits. Raptis et al. [23] suggested 
for the management of cancer target referral patients that web based software is effective in facilitating and improving the quality of information between users.

The Erasmus Medical Centre of Rotterdam developed an open structured data entry application to support structured recording of patient data in any medical domain. Roukema et al. [24] found that the electronic records of physical examination in paediatric outpatient care were more complete and contained additional information whereas in the patient history, which has mostly a narrative information nature, information was missing. Similar to NEOREG free text data entries are allowed to register findings which were not present as structured checkbox values. Some free text entry boxes could not be replaced by a structured entry. Configuration in NEOREG turns frequently occurring text entries into permanent checkbox values by adapting the variables and dynamically rebuilding the PDF questionnaire templates. Despite the increasing availability of electronic systems, clinicians often continue to use paper to complete their work. Saleem et al. [25] explored the factors that cause users to use papers instead of EHR. The technology should offer the same convenience in the clinical workflow as the usage of pen and paper to guarantee full adoption.

To guarantee system adoption, specific training of physicians will be required. Although the user interface is easy to use, previous implementations have already indicated that training is essential. Alberdi et al. [26] investigated the role of computerization in neonatal intensive care and found that a lack of system training affected the staff usage of the computerized information system for NICU. During the consultation of NEOREG on a client PC, we detected that users should be made aware of all functionality, their browser requirements and how to effectively fill out the PDF forms by demonstrating the usage. Additionally, documentation and a manual with the functionality are provided.

NEOREG Data may contribute to investigations of neonatal research networks. Fanaroff et al. [27] describe the changes in therapy practice and outcome amongst network centres during 15 years. The paper details the collected information of the Enice Kennedy Shriver National Institute of Child Health and Human Development (NICHD) Neonatal Research Network in the US. A very low birth weight (VLBW) registry has been maintained. VLBW incorporates perinatal and neonatal data of all infants with a birth weight lower than $1500 \mathrm{~g}$ cared for at the participating centres. Similar to NEOREG, maternal and infant data are collected and stored. 


\section{Conclusion}

The NEOREG system supports the registration of newborns infected with congenital cytomegalovirus. The web-based system replaces the existing paper-based forms with a similar electronic portable document forms. It allows remote access and telemonitoring of the patient's records. This paper describes the architecture and evaluation of the electronic registration system NEOREG. The database characteristics and performance of the NEOREG website have been thoroughly evaluated in this paper. When NEOREG electronic data records are used, data can be queried more easily to conduct clinical studies. It is expected that NEOREG electronic registration will contribute to higher data quality and detailed follow-up of patient registrations.

\section{Conflict of Interest}

The authors declare that they have no conflict of interest.

\section{References}

[1] Smets K, De Coen K, Dhooge I, Standaert L, Laroche S, Mahieu L et al. Selecting neonates with congenital cytomegalovirus infection for ganciclovir therapy. European Journal of Pediatrics 2006; 165:885-890.

[2] Naessens A, Casteels A, Wecatte L, Foulon W. A serologic strategy for detecting neonates at risk for congenital cytomegalovirus infection. Journal of Pediatrics 2005, 146:194-197.

[3] Perinatal activities in Flanders, Technical report 2010.

[4] Kimberlin DW, Lin C, Sanchez PJ, Weammler GJ, Dankner W, Shelton M et al. National Institute of Allergy and Infectious Diseases Collaborative Antiviral Study Group: Effect of ganciclovir therapy on hearing in symptomatic congenital cytomegalovirus disease involving the central nervous system: randomized controlled trial. Journal of Pediatrics 2003, 143:16-25.

[5] Smets K, Verrue N, Dhooge I. Implementation and results of bedside hearing screening with automated audiotry brainstem response in the neonatal intensive care unit. Acta Paediatrica 2012.

[6] Ford D, Jones K, Verplancke JP, Lyons R, John G, Brown G et al. The SAIL Databank: building a national architecture for e-health research and evaluation. BMC Health Services Research 2009, 9:157.

[7] Krishnan A, Nongkynrih B, Yadav K, Singh S, Gupta V. Evaluation of computerized health management information system for primary health care in rural India. BMC Health Services Research 2010, 10:310.

[8] Slagle TA, Gould JB. Database use in neonatal intensive care units: success or failure. Pediatrics 1992, 90(6):959-970. 
[9] McGregor C, Bryan G, Curry J, Tracy M. The E-baby Data Warehouse: A Case Study. Proceedings of the 35th Annual Hawaii International Conference on System Sciences (HICSS'02)-Volume 8, Washington, DC, USA: IEEE Computer Society 2002.

[10]Yang L, Frize M, Eng P, Walker RC, Catley C. Towards Ethical Decision Support and Knowledge Management in Neonatal Intensive Care 2004, 2:3420-3423.

[11]Skouroliakou M, Soloupis G, Gounaris A, Charitou A, Papasarantopoulos P, Markantonis SL, et al. Data analysis of the benefits of an electronic registry of information in a neonatal intensive care unit in Greece. Perspectives in health information management / AHIMA, American Health Information Management Association 2008, 5.

[12] Cordero L, Kuehn L, Kumar RR, Mekhjian HS. Impact of computerized physician order entry on clinical practice in a new-born intensive care unit. Journal of perinatology 2004, 24(2):88-93.

[13]Urschitz M, Lorenz S, Unterasinger L, Metnitz P, Preyer K, Popow C. Three years experience with a patient data management system at a neonatal intensive care unit. Journal of Clinical Monitoring and Computing 1998, 14:119-125.

[14]Steurbaut K, Van Hoecke S, Colpaert K, Lamont K, Taveirne K, Depuydt P et al. Use of web services for computerized medical decision support, including infection control and antibiotic management, in the intensive care unit. J Telemed Telecare 2010, 16:25-29.

[15]Steurbaut K, Hoecke SV, Taveirne K, Lamont K, De Turck F, Colpaert K et al. Design of Software Services for Computer-Based Infection Control and Antibiotic Management in the Intensive Care Unit. International Conference on eHealth, Telemedicine, and Social Medicine 2009, IEEE 2009:87-92.

[16]Pan H, Wang A, Jiang W. Discussion of course of E-commerce website construction based on java EE lightweight framework. 2nd International Conference on Education Technology and Computer (ICETC), 2010 1:442-445.

[17]Song B, Zhang Y, Zhou CS: Implementation on Network Teaching System Based on Java EE Architecture. Second International Conference on Information Technology and Computer Science (ITCS) 2010:227-231.

[18]De Backere F, Steurbaut K, De Turck F, Colpaert K, Decruyenaere J. On the Design of a Management Platform for Antibiotic Guidelines in the Intensive Care Unit. International Conference on Software Engineering Advances, 2010:406-411.

[19]Niziaek A, Zabierowski W, Napieralski A. Application of JEE 5 technologies for a system to support dental clinic management. Proceedings of International Conference on Modern Problems of Radio Engineering, Telecommunications and Computer Science, 2008:565-568.

[20]Migodzinski A, Ritter R, Kaminski M, Chlapinski J, Sakowicz B. Innovative data collecting system of services provided by medical laboratories. 11th International Conference The Experience of Designing and Application of CAD Systems in Microelectronics (CADSM) 2011:252-255, 
[21]Lowagie B. iText in Action, Second Edition. Manning Publications Co. October 2010.

[22]Christensen T, Grimsmo A. Instant availability of patient records, but diminished availability of patient information: a multi-method study of GP's use of electronic patient records. BMC medical informatics and decision making 2008(8):12.

[23]Raptis D, Graf R, Peck J, Mouzaki K, Patel V, Skipworth J et al. Development of an electronic webbased software for the management of colorectal cancer target referral patients. Informatics for Health and Social Care 2011, 36(3):117-131.

[24]Roukema J, Los RK, Bleeker SE, van Ginneken AM, van der Lei J, Moll HA. Paper Versus Computer: Feasability of an Electronic Medical Record in General Pediatrics. Pediatrics 117(1):15-21.

[25]Saleem JJ, Russ AL, Justice CF, Hagg H, Ebright PR, Woodbridge PA et al. Exploring the persistence of paper with the electronic health record. International journal of medical informatics 2009,78(9):618628.

[26]Alberdi E, Gilhooly K, Hunter J, Logie R, Lyon A, McIntosh N et al. Computerisation and Decision Making in Neonatal Intensive Care: A Cognitive Engineering Investigation. Journal of Clinical Monitoring and Computing 2000, 16(2):85-94.

[27]Fanaroff A. The NICHD neonatal research network: changes in practice and outcomes during the first 15 years. Seminars in Perinatology 2003, 27(4):281-287. 


\section{Figures}

Figure 1 - Transition from (a) the current paper-based workflow to (b) electronic records using NEOREG.

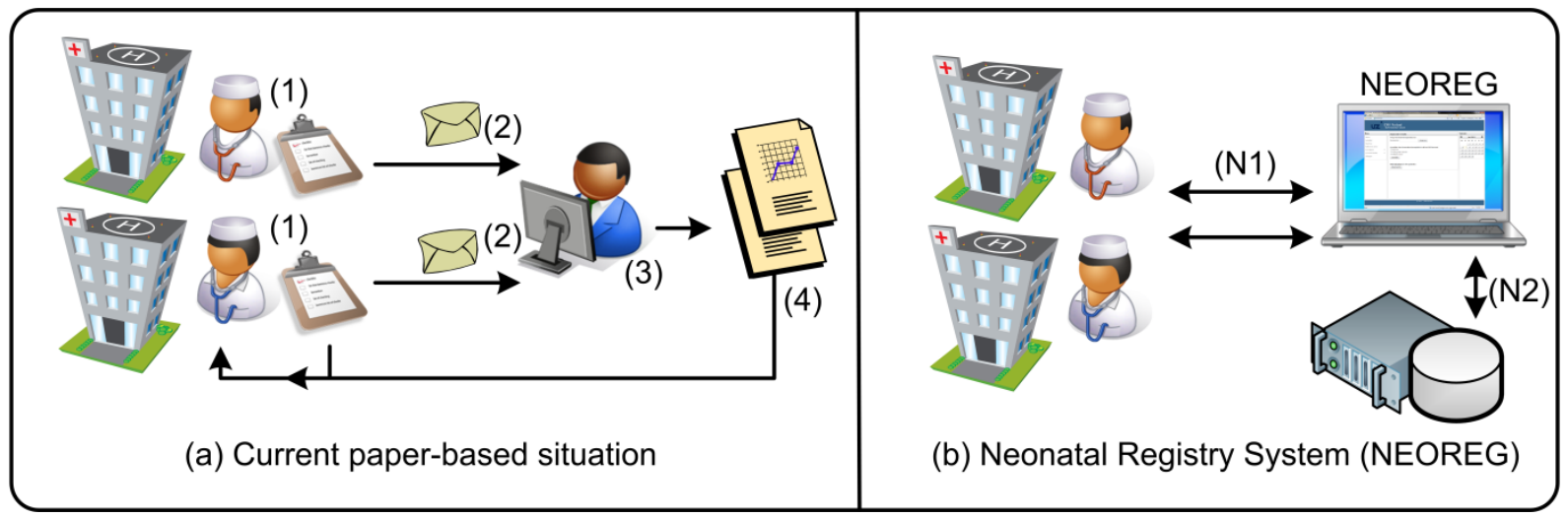


Figure 2 - The CMV Registration form gives an example of our CMV questionnaire including the text fields to register a newborn with cCMV.

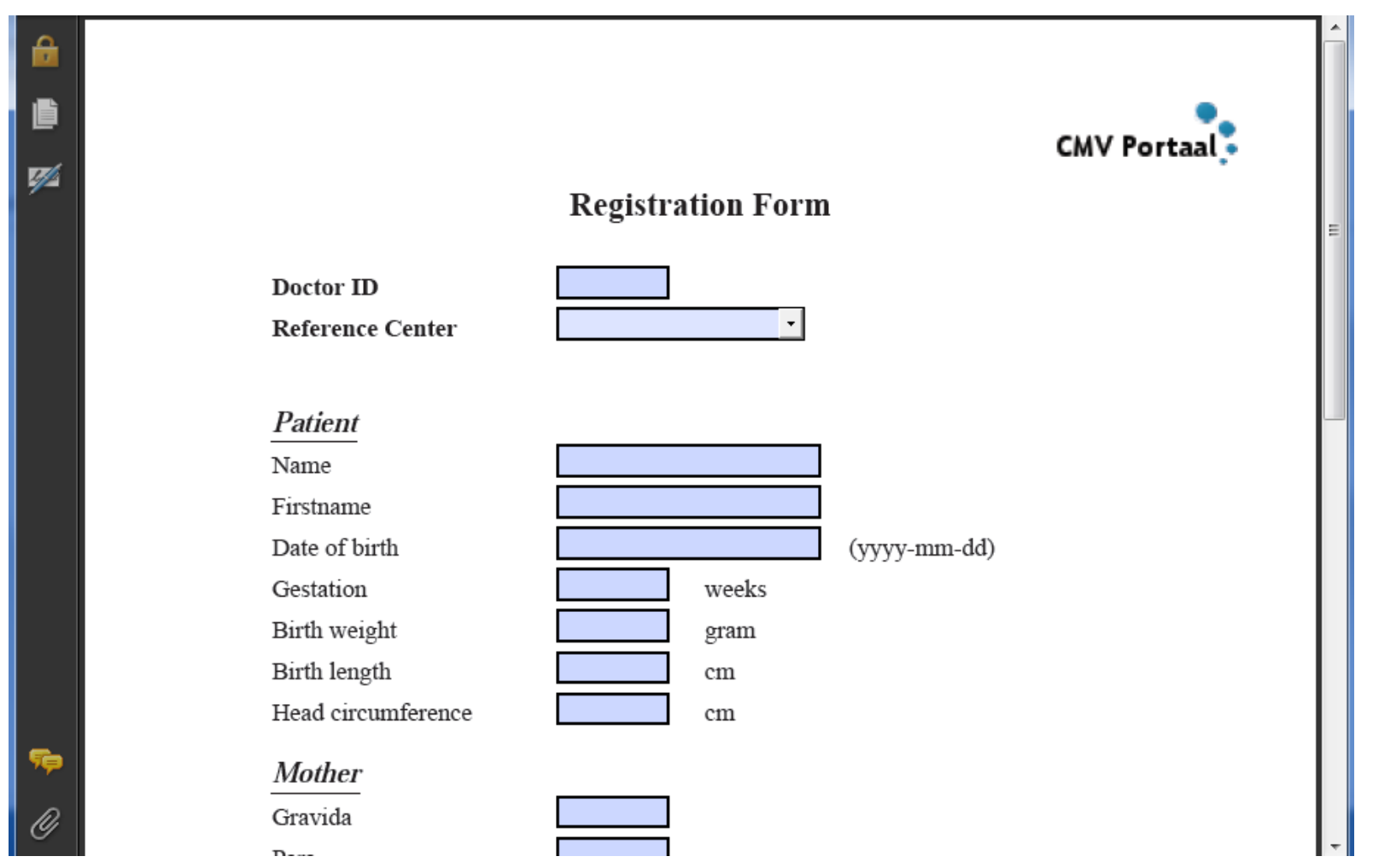


Figure 3 - High level overview of the NEOREG Layered Architecture.

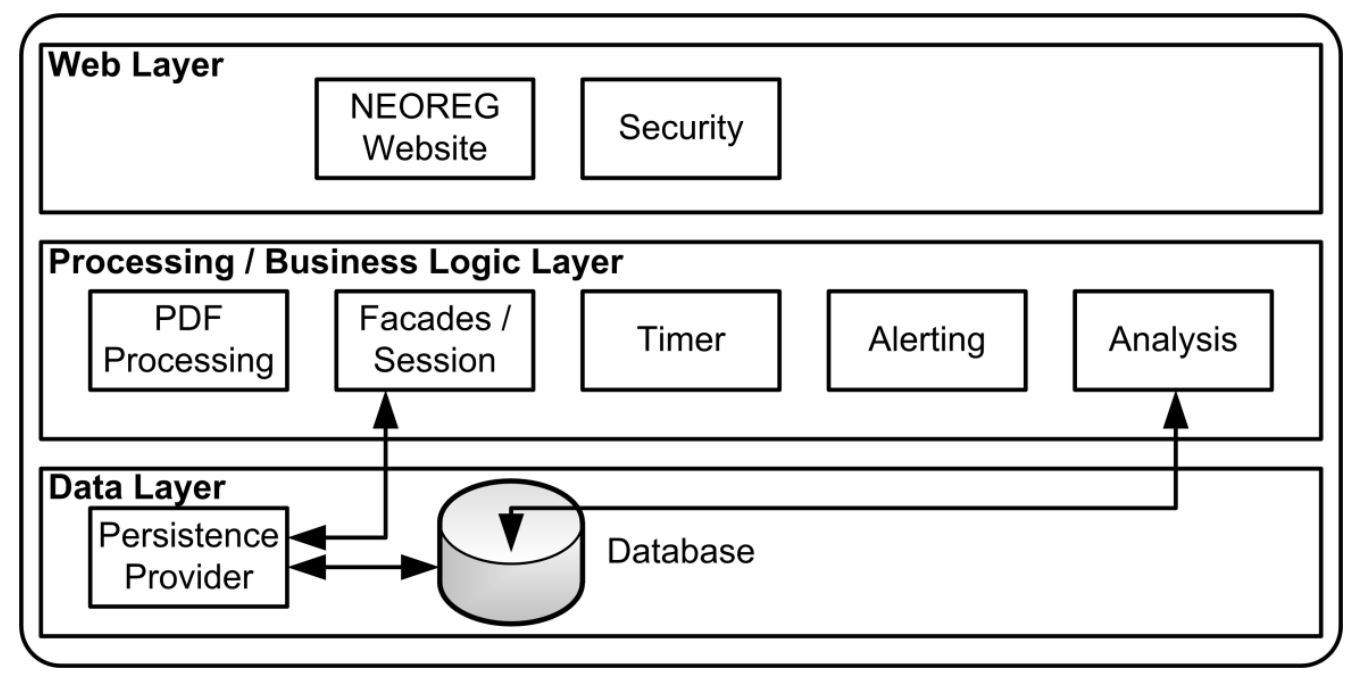


Figure 4 - Overview of the cCMV portal website. On the left hand side of the website, the physician can access the pages to register new forms, access previous data and check registered patients. On the right hand side a calendar pane is shown which is connected with the alerting component, to track follow-up of patients. The central pane contains a list of registration options for diagnostics or treatments. By adding options, the form is automatically changed and a new questionnaire template is produced.

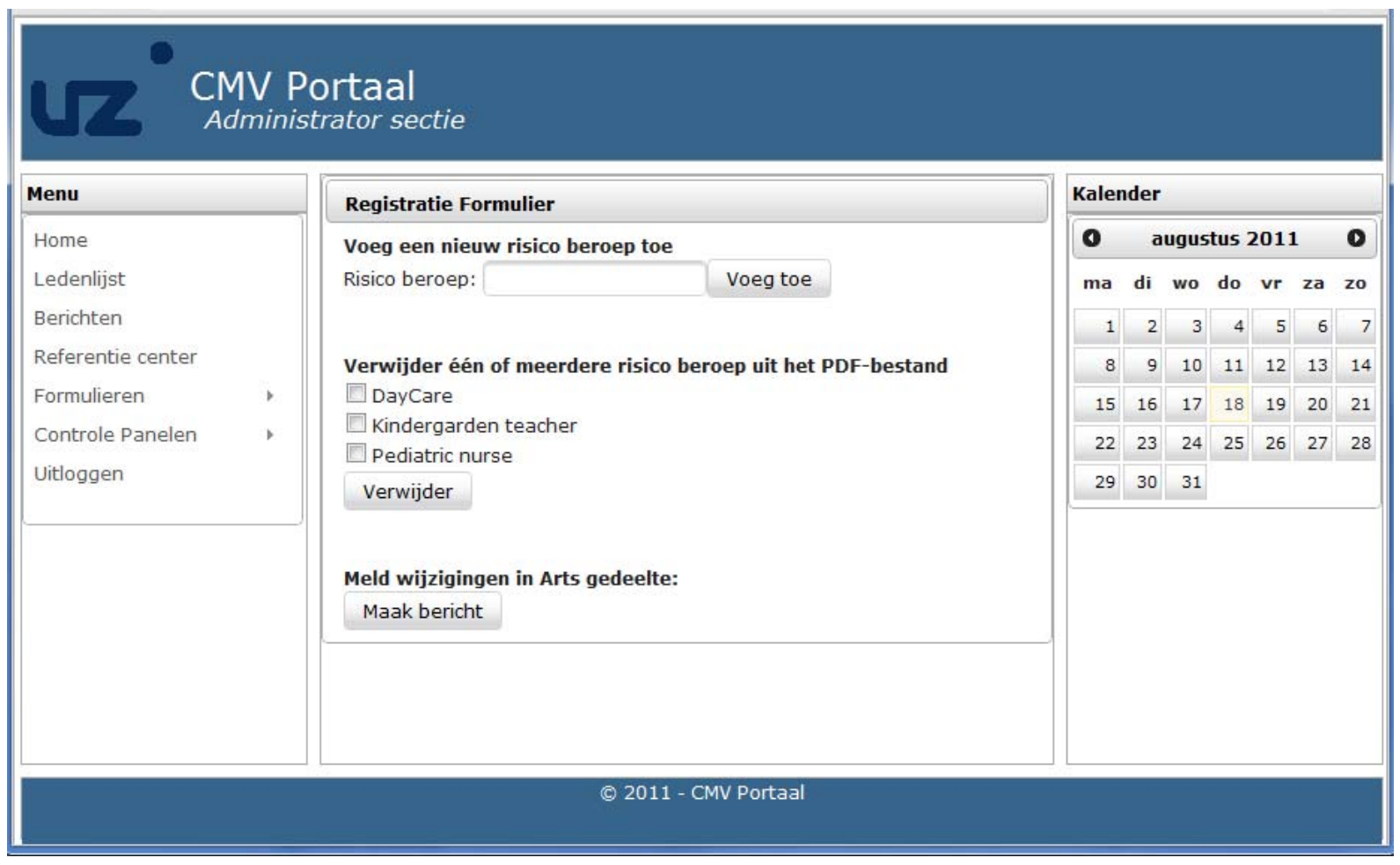


Figure 5 - Overview of the NEOREG database structure.

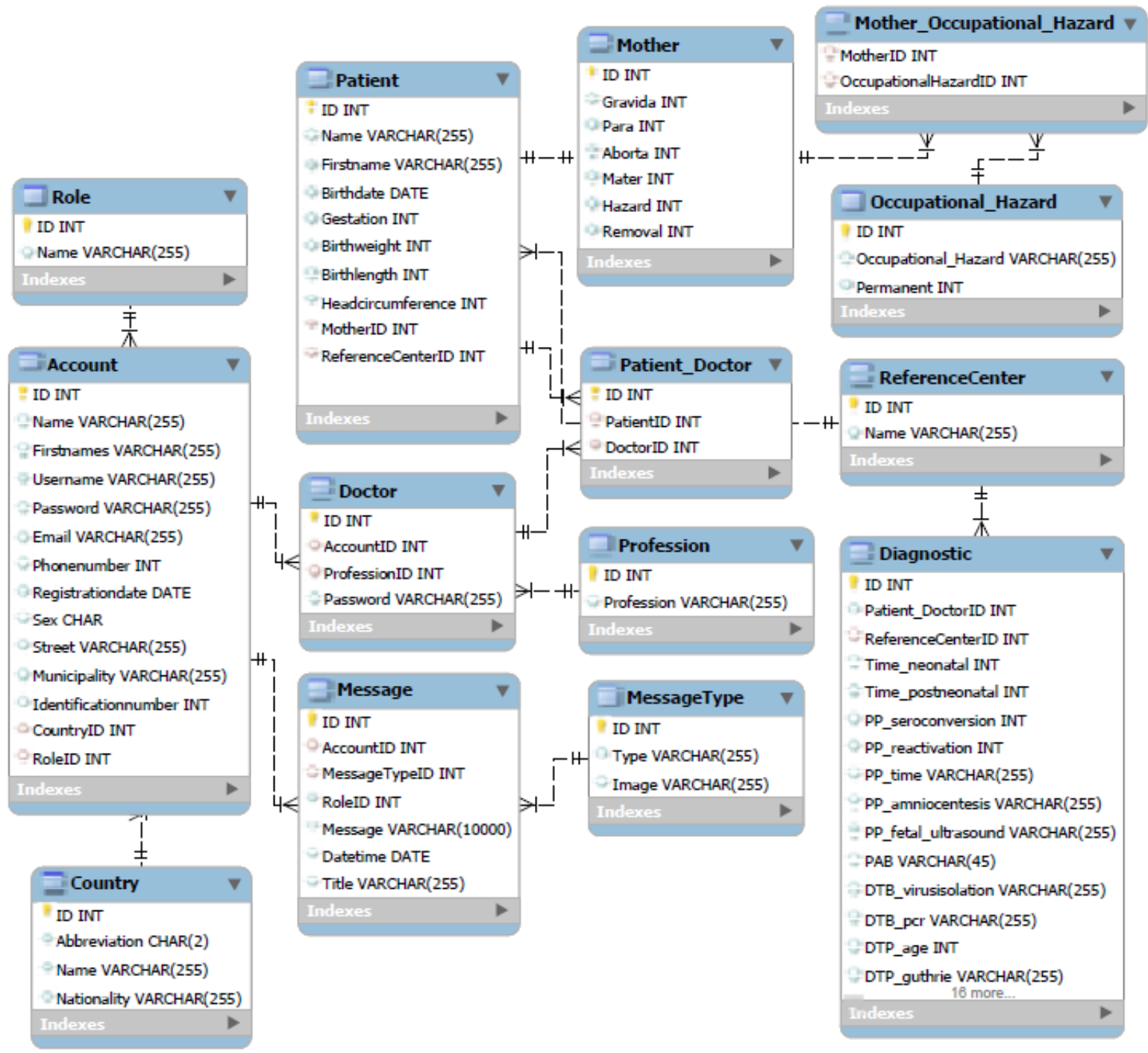


Figure 6 - Evaluation setup simulating (a) user requests and (b) data queries.

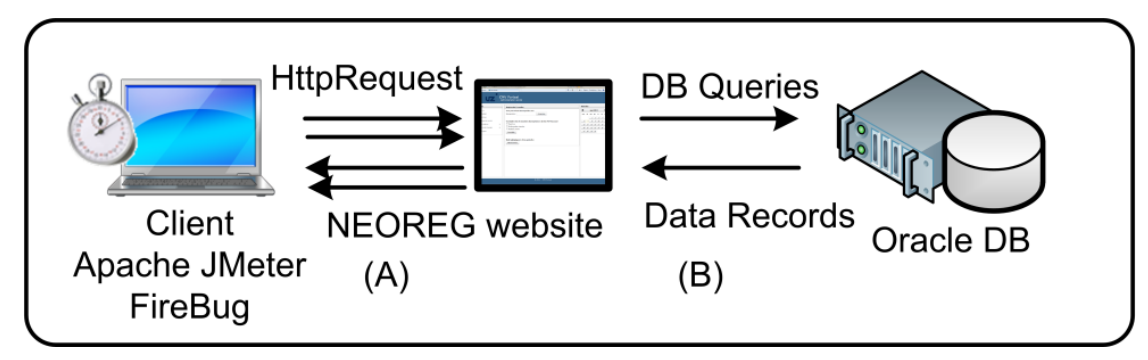


Figure 7 - Throughput and KB/sec with standard deviation.

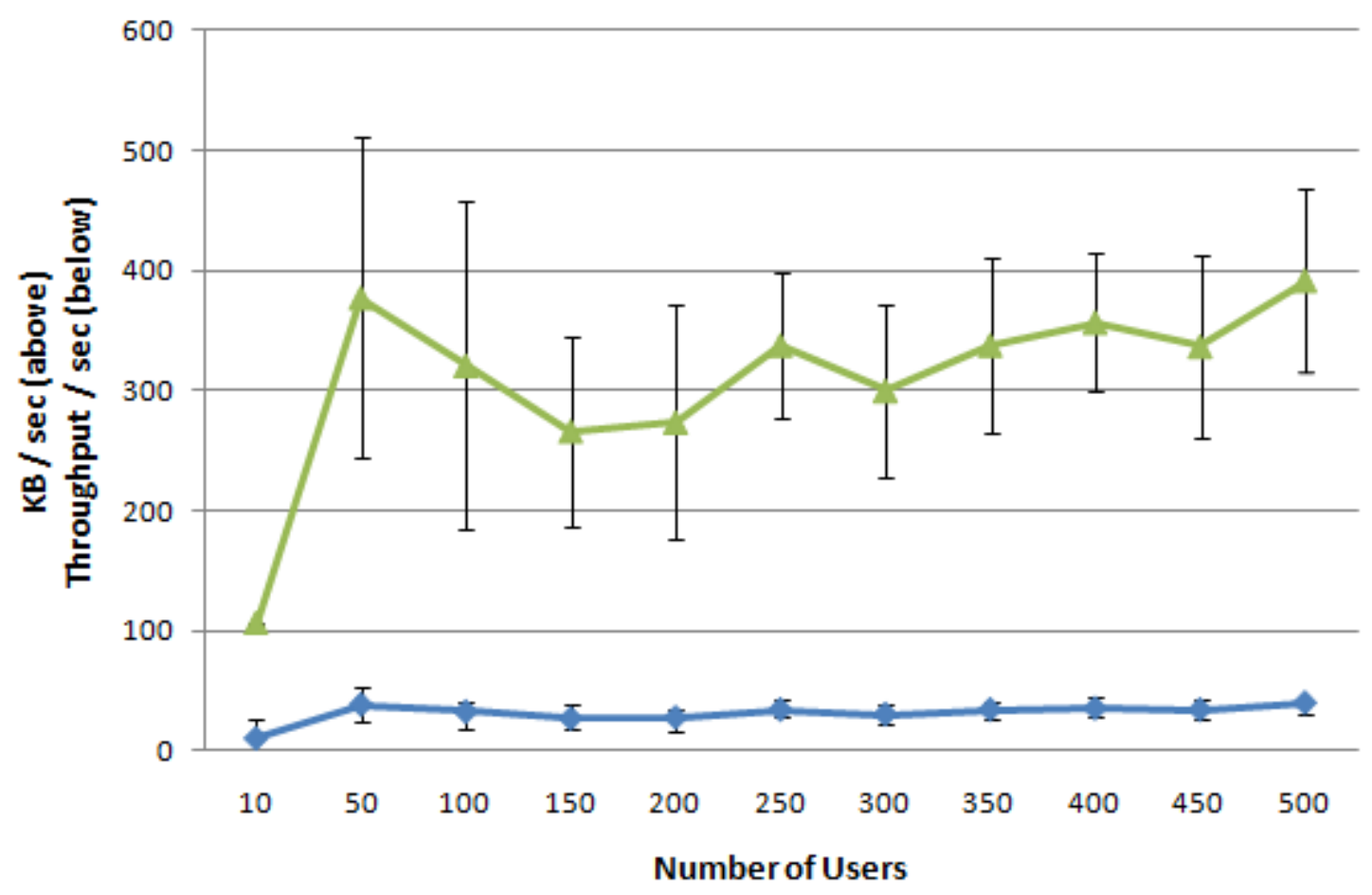


Figure 8 - Execution time of the data analysis cases.



\& Time Audiologic Data (s) Time Neurologic (s) 
Tables

Table 1 - Website performance measurements

\begin{tabular}{|l|r|r|r|}
\hline Webpage & Size (KB) & \multicolumn{1}{|c|}{ Time (s) } & \multicolumn{1}{|c|}{ Std dev (s) } \\
\hline Login page & 613.7 & 4.036 & 0.957 \\
\hline Physician overview & 623.4 & 3.319 & 1.031 \\
\hline Download page & 624.9 & 3.998 & 1.277 \\
\hline PDF selection page (registr) & 291.2 & 12.950 & 1.321 \\
\hline PDF selection page (follow) & 283.2 & 11.924 & 1.421 \\
\hline Patient list page & 670.8 & 5.534 & 1.853 \\
\hline Data analysis page & 807.7 & 6.564 & 1.515 \\
\hline
\end{tabular}

